\title{
ON EXPOSED POINTS OF THE RANGE OF A VECTOR MEASURE. II
}

\author{
R. ANANTHARAMAN
}

\begin{abstract}
If a weakly compact convex set $K$ in a real Banach space $X$ is strongly exposed by a dense set of functionals in $X^{\prime}$, it is proved that the functionals which expose $K$ form a residual set in $X^{\prime}$. If $\nu: \mathbb{Q} \rightarrow X$ is a measure, it follows that the set of exposing functionals of its range is a residual $G_{\delta}$ in $X^{\prime}$. This, in turn, is found to be equivalent to a theorem of B. Walsh on the residuality of functionals $x^{\prime} \in X^{\prime}$ for which $x^{\prime} \circ \nu \equiv \nu$.

If the set of exposed points of $\nu(Q)$ is weakly closed and $\nu_{A}$ is the restriction of $\nu$ to any set $A \in \mathbb{Q}$, it is further proved that every exposed point of the range of $\nu_{A}$ is of the form $\nu(A \cap E)$, where $E \in \mathbb{Q}$ and $\nu(E)$ is an exposed point of $\nu(\mathbb{Q})$.
\end{abstract}

1. Introduction. Throughout assume $X$ to be a real Banach space, $X^{\prime}$ the dual of $X$, and $\nu$ a measure defined on a $\sigma$-algebra $\mathcal{Q}$ of sets with values in $X$. As proved by Bartle, Dunford and Schwartz [3], there exists a finite positive measure $\lambda$ on $\mathbb{Q}$ such that $\nu \equiv \lambda$. As a refinement of this theorem, Rybakov [7] proved that there exists a functional $x^{\prime} \in X^{\prime}$ such that the signed measure $x^{\prime} \circ \nu \equiv \nu$. Such functionals have, in turn, been proved by B. Walsh [9] to form a residual $G_{\delta}$ set in $X^{\prime}$.

For any set $K$ in $X$, it may be recalled that a point $x \in K$ is an exposed point [5] of $K$ if there exists an $x^{\prime} \in X^{\prime}$ such that $x^{\prime}(x)>x^{\prime}(y)$ whenever $y \in K$, $y \neq x$. Such a functional $x^{\prime}$ is, in turn, said to expose the set $K$ at $x$. A point $x$ of $K$ is further called a strongly exposed point [5] of $K$ if there exists an $x^{\prime} \in X^{\prime}$ for which (i) $x^{\prime}(x)>x^{\prime}(y)$ whenever $y \in K, y \neq x$, and (ii) for every net $\left(x_{n}\right)$ in $K, x^{\prime}\left(x_{n}\right) \rightarrow x^{\prime}(x)$ implies that $x_{n} \rightarrow x$. The functional $x^{\prime}$ is then said to strongly expose $K$ at $x$. The set of exposed points of $K$ is denoted by $\exp K$, and, in case $K$ is convex, the set of its extreme points is denoted by ext $K$.

If the functionals $x^{\prime} \in X^{\prime}$ which strongly expose a weakly compact convex set $K$ in $X$ are dense in $X^{\prime}$, then the functionals $x^{\prime} \in X^{\prime}$ which expose $K$ are proved in Theorem 2 to be residual in $X^{\prime}$; moreover, if each functional that exposes $K$ is strongly exposing, then they form a residual $G_{\delta}$ set in $X^{\prime}$. This yields, in particular, the above theorem of $\mathrm{B}$. Walsh.

For every $A \in Q$, let $\nu_{A}$ denote the restriction of $\nu$ to $A$, viz. $\nu_{A}(E)$ $=\nu(A \cap E), E \in \mathbb{Q}$. In case of a finite-dimensional nonatomic measure $\nu$, Husain and Tweddle [4] proved that every extreme point of $\nu_{A}(\mathbb{Q})$ is of the form $\nu(A \cap E)$, where $E \in \mathbb{Q}$ and $\nu(E) \in$ ext $\nu(\mathbb{Q})$. If $\nu: \mathbb{Q} \rightarrow X$ is a measure such that the exposed points of its range form a weakly closed set, we obtain

Received by the editors August 29, 1974.

AMS (MOS) subject classifications (1970). Primary 28-XX; Secondary 28A45.

Key words and phrases. Exposed points, exposing functionals, range of vector measures, weakly compact convex sets. 
in Theorem 8 an analogous result for exposed points.

The author is deeply indebted to Professor K. M. Garg for his valuable guidance and help in the preparation of this paper.

2. Residuality of exposing functionals. Given a weakly compact convex set $K$ in $X$, we define, for every $x^{\prime} \in X^{\prime}$,

$$
K_{x^{\prime}}=\left\{x \in K: x^{\prime}(x)=\max x^{\prime}(K)\right\},
$$

and the map

$$
\rho_{K}: X^{\prime} \rightarrow R, \quad \rho_{K}\left(x^{\prime}\right)=\operatorname{diam} K_{x^{\prime}}, \quad x^{\prime} \in X^{\prime} .
$$

The author is grateful to the referee for an improvement in the proof of the following lemma.

LeMMA 1. For any weakly compact convex set $K$ in $X$, the map $\rho_{K}$ is continuous at every $x^{\prime} \in X^{\prime}$ which strongly exposes $K$.

Proof. Let $x^{\prime} \in X^{\prime}$ strongly expose $K$ at $x$. Then $\rho_{K}\left(x^{\prime}\right)=0$. Suppose that $\rho_{K}$ is not continuous at $x^{\prime}$. Then there exists $\varepsilon>0$, and a sequence $\left\{x_{n}^{\prime}\right\}$ in $X^{\prime}$ converging to $x^{\prime}$ for which $\rho_{K}\left(x_{n}^{\prime}\right) \geqslant \varepsilon$ for each $n$. Thus there exist, for each $n$, $a_{n}$ and $b_{n}$ in $K_{x_{n}^{\prime}}$ such that $\left\|a_{n}-b_{n}\right\| \geqslant \varepsilon / 2$.

Since $K$ is weakly compact, the sequence $\left\{a_{n}\right\}$ has a subnet $\left(a_{i}\right)$ which converges weakly to some point $a$ in $K$. Similarly, $\left(b_{i}\right)$ has a subnet $\left(b_{j}\right)$ which converges weakly to some $b \in K$. Now the set $K^{\prime}=\left\{x_{n}^{\prime} ; n \geqslant 1\right\} \cup\left\{x^{\prime}\right\}$ is norm-compact, and, as $K$ is bounded, the evaluation map from $K \times K^{\prime}$ to $R$, given by $\left(y, y^{\prime}\right) \rightarrow y^{\prime}(y), y \in K, y^{\prime} \in K^{\prime}$, is jointly continuous relative to the weak topology on $K$ and the norm topology on $K^{\prime}$. We thus have $x_{j}^{\prime}\left(a_{j}\right)$ $\rightarrow x^{\prime}(a)$. If $\beta=\sup x^{\prime}(K)$ and $\beta_{j}=\sup x_{j}^{\prime}(K)$ for every $j$, then obviously $\beta_{j} \rightarrow \beta$. Since $a_{j} \in K_{x_{j}^{\prime}}$ for each $j, x_{j}^{\prime}\left(a_{j}\right)=\beta_{j}$, and so we have $x_{j}^{\prime}\left(a_{j}\right) \rightarrow \beta$. Thus we obtain $x^{\prime}(a)=\lim x_{j}^{\prime}\left(a_{j}\right)=\beta=x^{\prime}(x)$. Since $\left(a_{j}\right) \rightarrow a$ weakly, we have $x^{\prime}\left(a_{j}\right) \rightarrow x^{\prime}(a)$. As $x^{\prime}$ strongly exposes $K$ at $x$, we obtain $a=x$ and $\left\|a_{j}-x\right\| \rightarrow 0$.

By a similar argument, one obtains $x^{\prime}(b)=\beta, b=x$ and $\left\|b_{j}-x\right\| \rightarrow 0$, so that $\left\|a_{j}-b_{j}\right\| \rightarrow 0$, contrary to the choice of the $a_{n}$ 's and $b_{n}$ 's. Hence $\rho_{K}$ is continuous at $x^{\prime}$.

REMARK. It is clear from the above proof that $\rho_{K}$ is upper semicontinuous when $K$ is norm-compact. It would be interesting to investigate weaker hypotheses on $K$ under which $\rho_{K}$ is upper semicontinuous.

THEOREM 2. If the set of strongly exposing functionals of a weakly compact convex set $K$ in $X$ is dense in $X^{\prime}$, then the set of its exposing functionals is residual in $X^{\prime}$.

In case every exposing functional of $K$ is further strongly exposing, then they form a residual $G_{\delta}$ set in $X^{\prime}$.

Proof. Let $C$ denote the set of points of continuity of $\rho_{K}$, and let $X_{e}^{\prime}$ and $X_{s}^{\prime}$ denote the sets of functionals $x^{\prime} \in X^{\prime}$ which expose or strongly expose $K$ respectively. Since $X_{s}^{\prime}$ is, by hypothesis, dense in $X^{\prime}$, and $\rho_{K}$ vanishes at every point of $X_{s}^{\prime} \rho_{K}$ is zero at every point where it is continuous. But then $x^{\prime}$ exposes 
$K$ whenever $\rho_{K}\left(x^{\prime}\right)=0$. We thus have, with the help of Lemma $1, X_{s}^{\prime} \subset C$ $\subset X_{e}^{\prime}$.

Since the points of continuity of any real valued function form a $G_{\delta}$ set, $X_{e}^{\prime}$ contains the dense $G_{\delta}$ set $C$, and so $X_{e}^{\prime}$ is residual in $X^{\prime}$. In case $X_{e}^{\prime} \subset X_{s}^{\prime}$, we have $X_{e}^{\prime}=C$, whence the second part of the theorem.

In case $\nu: \mathbb{Q} \rightarrow X$ is a measure, the set $K=\overline{\text { co }} \nu(\mathbb{Q})$ is weakly compact by a theorem of Bartle, Dunford and Schwartz [3]. Hence there exists, according to Theorem 4 of Amir and Lindenstrauss [1], at least one $x^{\prime} \in X^{\prime}$ which exposes $K$. For any other $y^{\prime} \in X^{\prime}$ it follows from Theorems 2 and 4 of [2] and Rybakov [7] that all but countably many elements in the segment from $x^{\prime}$ to $y^{\prime}$ expose $K$, and so $X_{e}^{\prime}$ is dense in $X^{\prime}$. But $X_{e}^{\prime} \subset X_{s}^{\prime}$ as it follows from the proof of Theorem 5 of [2], and so we have

COROLlaRY 3. If $\nu$ is a measure with values in $X$, the exposing functionals of its range form a residual $G_{\delta}$ set in $X^{\prime}$.

According to Theorems 2 and 4 of [2], an $x^{\prime} \in X^{\prime}$ exposes $K$ if and only if $x^{\prime} \circ \nu \equiv \nu$, and so the above corollary yields in turn

COROLLARY 4 (WALSH [9]). If $\nu$ is a measure with values in $X$, the functionals $x^{\prime} \in X^{\prime}$, for which $x^{\prime} \circ \nu \equiv \nu$, form a residual $G_{\delta}$ set in $X^{\prime}$.

3. Exposed points of the range of a restriction of a measure. If $A$ and $B$ are two weakly compact convex sets in $X$, in analogy with the definition of $\operatorname{ext}_{B} A$ in [4], we define $\exp _{B} A$ to be the set of those exposed points $x$ of $A$ for which there exists some exposed point $y$ of $B$ such that $x+y \in \exp (A+B)$.

Proposition 5. If $A$ and $B$ are two weakly compact convex sets in $X$, then $\exp _{B} A$ is weakly dense in $\exp A$.

Proof. By using Theorem 4 of Amir and Lindenstrauss [1] in place of the Krein-Mil'man Theorem in the proof of Theorem 1 of [4], it may be verified that $\exp _{B} A$ is weakly dense in ext $A$, and since $\exp _{B} A \subset \exp A$ and $\exp A$ $C$ ext $A$, as is well known, it follows that $\exp _{B} A$ is weakly dense in $\exp A$.

On using Corollary 7 of Troyanski [8] instead, the above proposition is found to hold for the set of strongly exposed points as well.

LEMMA 6. If $\nu: \mathbb{Q} \rightarrow X$ is a measure, then the weak and norm topologies coincide on the set of extreme points of the closed convex hull of $\nu(\mathbb{Q})$.

Proof. There exists a finite positive measure $\lambda$ on $\mathbb{Q}$ such that $\nu \equiv \lambda$ (see [3]). For each $\phi \in L_{\infty}(\lambda)$, its Bartle-Dunford-Schwartz integral [3] $\int \phi d \nu$ $\in X$. Let $T: L_{\infty}(\lambda) \rightarrow X$ be defined by $T(\phi)=\int \phi d \nu, \phi \in L_{\infty}(\lambda)$, and let $T_{\nu}$ denote the restriction of $T$ to the set $P=\left\{\phi \in L_{\infty}(\lambda): 0 \leqslant \phi \leqslant 1 \lambda\right.$-a.e. $\}$. It follows from the Radon-Nikodým theorem that $T$ is continuous relative to the weak*-topology $\sigma\left(L_{\infty}(\lambda), L_{1}(\lambda)\right)$ on $L_{\infty}(\lambda)$ and the weak topology on $X$. According to a theorem of Bartle, Dunford and Schwartz [3], the set $K=\overline{\mathrm{co}} \nu(\mathbb{Q})$ is weakly compact, and so we have $T_{\nu}(P)=K$ (see, e.g., [2, Lemma 1]).

We need to show that the weak topology on ext $K$ is finer than the norm topology. Let $\left(x_{i}\right)$ be a net in ext $K$ converging weakly to an element $x$ in ext $K$. Then there exist, by Proposition 2 of [2], unique sets $E_{i}, E \in \mathbb{Q}$, such 
that $T_{\nu}^{-1}\left(x_{i}\right)=\left\{\chi_{E_{i}}\right\}$ for each $i$ and $T_{\nu}^{-1}(x)=\left\{\chi_{E}\right\}$, where $\chi_{A}$ denotes the characteristic function of $A \in \mathcal{Q}$. It may easily be verified that $P$ is weak*compact, and since the net $\left(\chi_{E_{i}}\right)$ is contained in $P$, there exists a subnet $\left(\chi_{E_{j}}\right)$ of $\left(\chi_{E_{i}}\right)$ converging to some $\phi \in P$ relative to the weak*-topology. As $T_{\nu}:\left(P, w^{*}\right) \rightarrow(K, w)$ is continuous, $\left(T_{\nu}\left(\chi_{E_{j}}\right)\right) \stackrel{w}{\longrightarrow} T_{\nu}(\phi)$, i.e. $\left(x_{j}\right) \stackrel{w}{\longrightarrow} T_{\nu}(\phi)$, and since $\left(x_{j}\right) \stackrel{w}{\longrightarrow} x$, we have $T_{\nu}(\phi)=x=T_{\nu}\left(\chi_{E}\right)$, and so $\phi=\chi_{E}$ (by [2, Proposition 2]). Now the weak*-topology coincides with the $L_{1}(\lambda)$-norm topology on the set of characteristic functions, and so $\left\|\chi_{E_{j}}-\chi_{E}\right\|_{1} \rightarrow 0$. On identifying $Q$ with the above subset of $L_{1}(\lambda)$, since $\nu: \mathbb{Q} \rightarrow X$ is continuous relative to the $L_{1}(\lambda)$-norm topology on $Q$ and the norm topology on $X$, we have $\| \nu\left(E_{j}\right)$ $-\nu(E) \| \rightarrow 0$. Thus every weakly convergent net in ext $K$ has a subnet that converges in the norm. Hence the lemma.

Lemma 7. If $\nu: \mathbb{Q} \rightarrow X$ is a measure, then for every $A \in \mathbb{Q}$ we have

$$
\begin{aligned}
\{\nu(E \cap A): \nu(E) \in \exp \nu(\mathbb{Q})\} & \subset \exp \nu_{A}(\mathbb{Q}) \\
& \subset\{\nu(E \cap A): \nu(E) \in \exp \nu(\mathbb{Q})\}^{-} .
\end{aligned}
$$

Proof. Let $K=\overline{\operatorname{co}} \nu(\mathbb{Q}), K_{1}=\overline{\operatorname{co}} \nu_{A}(\mathbb{Q})$ and $K_{2}=\overline{\operatorname{co}} \nu_{A^{c}}(\mathbb{Q})$, where $A^{c}$ denotes the complement of $A$. According to [2, Theorem 4], we have $\exp \nu(\mathbb{Q})=\exp K$ and $\exp \nu_{A}(\mathbb{Q})=\exp K_{1}$. It is easy to see that $K=K_{1}$ $+K_{2}$, and that an $x^{\prime} \in X^{\prime}$ exposes $K$ at $\nu(E)$ if and only if $x^{\prime}$ exposes $K_{1}$ at $\nu(E \cap A)$ and $K_{2}$ at $\nu\left(E \cap A^{c}\right)$, whence we have $\exp _{K_{2}} K_{1}=\{\nu(E \cap A): \nu(E)$ $\in \exp K\}$. The lemma now follows from Proposition 5 and Lemma 6.

TheOREM 8. If $\nu: \mathbb{Q} \rightarrow X$ is a measure such that $\exp \nu(\mathbb{Q})$ is weakly closed, then for every $A \in \mathbb{Q}$ we have

$$
\exp \nu_{A}(\mathscr{Q})=\{\nu(E \cap A): \nu(E) \in \exp \nu(\mathscr{Q})\}
$$

Proof. According to Lemma 7, it suffices to prove that the set on the righthand side is norm-closed. Let $\nu\left(E_{n}\right) \in \exp \nu(Q)$ for each $n$, and assume that the sequence $\left\{\nu\left(E_{n} \cap A\right)\right\}$ converges to $x \in X$. As $\chi_{E_{n}} \in P$ for each $n$ and $(P$, $\left.w^{*}\right)$ is compact, there exists a subnet $\left(\chi_{E_{m}}\right)$ of $\left\{\chi_{E_{n}}\right\}$ and some $\phi \in P$ such that $\chi_{E_{m}} \stackrel{w^{*}}{\longrightarrow} \phi$. Since $T_{\nu}:\left(P, w^{*}\right) \rightarrow(K, w)$ is continuous, the net $\left(T_{\nu}\left(\chi_{E_{m}}\right)\right)$ $\stackrel{w}{\longrightarrow} T_{\nu}(\phi)$, i.e. $\left(\nu\left(E_{m}\right)\right) \stackrel{w}{\longrightarrow} T_{\nu}(\phi)$. As $\nu\left(E_{m}\right) \in \exp \nu(\mathbb{Q})$ for each $m$ and $\exp \nu(\mathscr{Q})$ is weakly closed, we have $T_{\nu}(\phi) \in \exp \nu(\mathscr{Q})(\subset$ ext $K)$, and so, by Proposition 2 of [2] there exists a unique set $E \in \mathbb{Q}$ such that $\phi=\chi_{E}$. But then $\chi_{E_{m}} \stackrel{w^{*}}{\longrightarrow} \chi_{E}$ and we obtain, as in the proof of Lemma 6, $\left\|\chi_{E_{m}}-\chi_{E}\right\|_{1}$ $\rightarrow 0$, whence it follows that $\left\|\chi_{E_{m} \cap A}-\chi_{E \cap A}\right\|_{1} \rightarrow 0$. Since $\nu: \mathbb{Q} \rightarrow X$ is continuous, the net $\left(\nu\left(E_{m} \cap A\right)\right)$ converges to $\nu(E \cap A)$. By hypothesis, $\left\|\nu\left(E_{m} \cap A\right)-x\right\| \rightarrow 0$, and so $x=\nu(E \cap A)$. Since $\nu(E) \in \exp \nu(\mathbb{Q})$, this completes the proof.

REMARK. When exp $\nu(\mathscr{Q})$ is not closed, the above theorem does not hold, in general, even for a finite-dimensional measure. For, as proved by Rickert [6, Theorem 1], there exists a nonatomic measure $\nu_{1}$ defined on the $\sigma$-algebra of Borel subsets of $A=[0,1]$ whose range is the closed unit disk in $R^{2}$. Let $\nu_{2}$ be the Lebesgue measure on the Borel subsets of $B=[1,2]$ whose range is the 
segment from $(0,0)$ to $(1,0)$. Let $\mathscr{Q}$ be the $\sigma$-algebra of Borel subsets of [0,2], and define the measure $\nu: \mathbb{Q} \rightarrow R^{2}$ by $\nu(E)=\nu_{1}(E \cap A)+\nu_{2}(E \cap B), E \in \mathbb{Q}$. The range of $\nu$ is the convex hull of the two disks with radius unity and centers $(1,0)$ and $(0,0)$. The point $(0,1)$ is an exposed point of $\nu_{A}(\mathbb{Q})$, and it may easily be verified that $(0,1)$ is not of the form $\nu(E \cap A)$ for any exposed point $\nu(E)$ of the range of $\nu$. We do not know if Theorem 8 is true when $\exp \nu(\mathbb{Q})$ is only norm-closed.

\section{REFERENCES}

1. D. Amir and J. Lindenstrauss, The structure of weakly compact sets in Banach spaces, Ann. of Math. (2) 88 (1968), 35-46. MR 37 \#4562.

2. R. Anantharaman, On exposed points of the range of a vector measure, Proc. Conf. on Vector Measures (Snowbird, Utah), Academic Press, New York, 1973, pp. 7-22.

3. R. G. Bartle, N. Dunford and J. T. Schwartz, Weak compactness and vector measures, Canad. J. Math. 7 (1955), 289-305. MR 16, 1123.

4. T. Husain and I. Tweddle, On the extreme points of the sum of two compact convex sets, Math. Ann. 188 (1970), 113-122. MR 42 \#6571.

5. J. Lindenstrauss, On operators which attain their norm, Israel J. Math. 1 (1963), 139-148. MR 28 \#3308.

6. N. W. Rickert, Measures whose range is a ball, Pacific J. Math. 23 (1967), 361-371. MR 36 \#5296.

7. V. I. Rybakov, Theorem of Bartle, Dunford and Schwartz concerning vector measures, Mat. Zametki 7 (1970), 247-254 = Math. Notes 7 (1970), 147-151. MR 41 \#5591.

8. S. L. Trojanski, On locally uniformly convex and differentiable norms in certain non-separable Banach spaces, Studia Math. 37 (1970/71), 173-180. MR 46 \# 5995.

9. B. J. Walsh, Mutual absolute continuity of sets of measures, Proc. Amer. Math. Soc. 29 (1971), 506-510. MR 43 \#4998.

Department of Mathematics, University of Alberta, Edmonton T6G 2GL, Alberta, CANADA 